\title{
Wall accumulation of bacteria with different motility patterns
}

\author{
Paolo Sartori, ${ }^{*}$ Enrico Chiarello, Gaurav Jayaswal, Matteo Pierno, ${ }^{\dagger}$ and Giampaolo Mistura \\ Dipartimento di Fisica e Astronomia “Galileo Galilei,” Università di Padova, via Marzolo 8, 35131 Padova PD, Italy \\ Paola Brun \\ Dipartimento di Medicina Molecolare, Università di Padova, via Gabelli 63, 35121 Padova PD, Italy \\ Adriano Tiribocchi and Enzo Orlandini ${ }^{\ddagger}$ \\ Dipartimento di Fisica e Astronomia “Galileo Galilei," Università di Padova and INFN, via Marzolo 8, 35131 Padova PD, Italy
}

(Received 6 December 2017; revised manuscript received 31 January 2018; published 13 February 2018)

\begin{abstract}
We systematically investigate the role of different swimming patterns on the concentration distribution of bacterial suspensions confined between two flat walls, by considering wild-type motility Escherichia coli and Pseudomonas aeruginosa, which perform Run and Tumble and Run and Reverse patterns, respectively. The experiments count motile bacteria at different distances from the bottom wall. In agreement with previous studies, an accumulation of motile bacteria close to the walls is observed. Different wall separations, ranging from 100 to $250 \mu \mathrm{m}$, are tested. The concentration profiles result to be independent on the motility pattern and on the walls' separation. These results are confirmed by numerical simulations, based on a collection of self-propelled dumbbells-like particles interacting only through steric interactions. The good agreement with the simulations suggests that the behavior of the investigated bacterial suspensions is determined mainly by steric collisions and self-propulsion, as well as hydrodynamic interactions.
\end{abstract}

DOI: 10.1103/PhysRevE.97.022610

\section{INTRODUCTION}

Many unicellular organisms (e.g., bacteria, algae) live in aqueous media and actively swim by converting free energy from the environment into work $[1,2]$. Due to their micrometric size, the dynamics is characterized by both low Reynolds numbers [3-6] and thermal fluctuations [7-9], so that they can be roughly considered as a suspension of self-propelled colloids or active Brownian particles [10,11]. Motility is a fundamental property of many bacteria. Indeed, it constitutes a competitive advantage to improve colonizing ability, penetrate mucus layers, enhance pathogenicity, access nutrient-rich niches, and increase bacterial growing rate [12]. The motility behavior near surfaces is of particular interest since very often bacteria can be found in geometrically confined environments (e.g., wet soil interstices, host living tissues, filters) that can alter their motility and spatial distribution [13-18]. For instance, Escherichia coli bacteria are observed to swim in clockwise circular trajectories close to liquid-solid interfaces $[19,20]$ and counterclockwise close to liquid-air interfaces [21]. Another example is the accumulation of swimming microorganisms close to a solid surface, an effect that has been observed and studied by many authors since the 1960s [22-27] but for which a general consensus on the possible driving mechanisms is still lacking. In particular, it is still under debate to which extent the bacteria density profile in the proximity of the wall

\footnotetext{
*paolo.sartori.3@unipd.it

${ }^{\dagger}$ matteo.pierno@unipd.it

${ }^{\ddagger}$ orlandini@pd.infn.it
}

depends either on hydrodynamic interactions [23,27] or on a combination of steric collisions with the wall and rotational diffusion $[24,25,28]$. A possible source of complexity is the wide variety of bacteria, each with its own size, shape, and motion mechanism that may give rise to detectable differences in bacteria accumulation at the walls [26].

Arguably, the most common bacterium used in experiments of microswimmers' motility near surfaces is E. coli, a multiflagella swimmer with cylindrical-shaped body of size about $1 \times 2 \mu \mathrm{m}$ [29-31]. The motion of the wild-type strains of $E$. coli can be described by a sequence of straight trajectories (runs), with average speed on the order of $20-30 \mu \mathrm{m} / \mathrm{s}$, alternated by fast and erratic rotation of the cell (tumbles), occurring with a frequency of about $1 \mathrm{~Hz}$ and an average angle $\simeq 70^{\circ}$ [32-34] [see Fig. 1(a)]. Runs correspond to counterclockwise rotation of all flagella forming a bundle, while tumbles occur when one or more flagella temporary switch to clockwise rotation and the bundle separates [35]. For this bacterium, the interplay between the random reorientation of the tumbling mechanism and the rectifying action of the surface is not yet completely understood [24,26,36], especially if compared with its most studied mutant counterpart where the Run and Tumble mechanism is replaced by a smooth swimming motion [23-25,27-29]. Another class of microswimmers, with similar body shape and dimensions, are the singly flagellated bacteria such as Pseudomonas aeruginosa that perform a back and forth (Run and Reverse) motion [32,37] [see Fig. 1(b)] depending on the rotational direction (clockwise or anticlockwise) of the flagellum [38], which switches with a frequency of about $1 \mathrm{~Hz}$.

Although different swimming patterns and cell shapes can impact differently on the accumulation properties of 
(a) E. coli

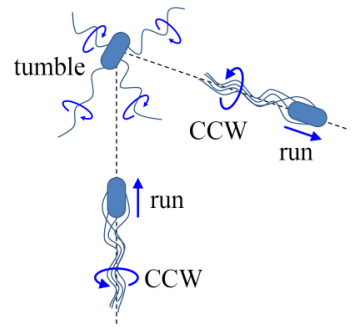

(d)

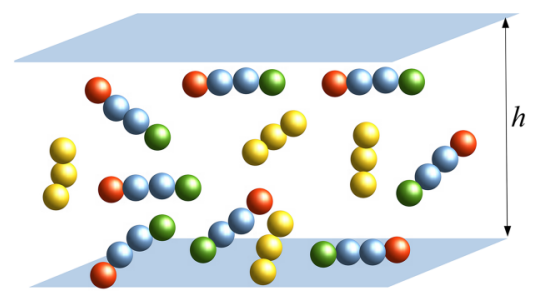

(c)

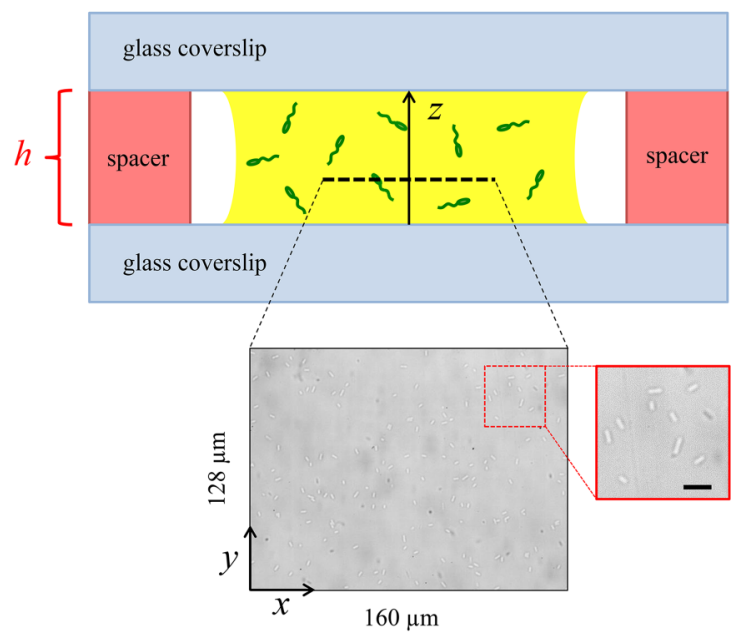

FIG. 1. (a), (b) Swimming patterns: Run and Tumble of E. coli (a); Run and Reverse of P. aeruginosa (b). (c) Sketch of the experimental setup: a drop of the bacterial suspension is closed between two glass coverslips with separation $h$, and it is observed in a bright field along the $z$ direction from below with focal plane $(x, y)$ parallel to the walls. The scale bar in the zoom of the field of view corresponds to $5 \mu \mathrm{m}$. (d) Sketch of the model used in the simulations: for illustration purposes, we plot bodies of different anisotropy: the multicolored ones are made by $M=4$ beads and are characterized by a head (red) and a tail (green) bead. The active force $F_{a}$ is applied to each bead pointing along the tail-head direction. The yellow bodies are less anisotropic $(M=3)$. In this case no head-tail direction is highlighted since these bodies describe nonmotile cells $\left(F_{a}=0\right)$. Note that our simulations consider active and passive bodies with the same anisotropy (same $M$ ).

bacteria near surfaces, to our knowledge no experimental work addresses this point systematically and within the same investigational framework.

Here we present a joint experimental and computational study of the tendency of bacteria with different swimming patterns to aggregate in proximity of rigid walls. Experiments are performed on bacterial suspensions of $E$. coli and $P$. aeruginosa that display, respectively, Run and Tumble and Run and Reverse motion. The experimental measurements of the density profile of the suspension across the confining region are compared with the results of simulations based on a simple model of self-propelled particles in which just a few physical properties, such as the cell anisotropy, the cell-cell and wall-cell steric interactions, and the roto-translational Brownian motion of each cell due to thermal fluctuations, are considered. Being very simple and generic, the model can be easily adapted to describe different motion mechanisms and, specifically, self-propelled cells performing Run and Tumble and Run and Reverse motion. The agreement between the experimental findings and the simulations suggests that the behavior of the investigated bacterial suspensions is determined mainly by steric collisions and self-propulsion, in addition to hydrodynamic interactions.

The paper is organized as follows: in the first and second parts of Sec. II we describe the preparation of the bacterial suspensions and the setup for the experiments, including the imaging techniques used to estimate the density profiles, respectively. The third part is devoted to the numerical model. Section III is the core of the paper with the presentation of the experimental findings and their comparison with the simulation results. For a more complete overview of the problem we also present additional simulations by varying the values of parameters such as the slit width, the cell velocity and anisotropy, and the density of the suspensions in ranges not easily accessible to experiments. Finally, the last section is devoted to a summary of the main results and to conclusions.

\section{MATERIALS AND METHODS}

The samples consist of active suspensions of $E$. coli strain MG1655 (LGC Standards; Milan, Italy) or wild-type P. aeruginosa (isolated from a clinical specimen and identified by mass spectrometry). The E. coli MG1655 strain has an insertion element in the genomic region regulating the transcription of flagellar genes encoding four or more flagella and making this strain hypermotile as compared with the wild-type strains [39]. E. coli is grown at $30^{\circ} \mathrm{C}$, shaken at $150 \mathrm{rpm}$ in Lysogeny broth (1\% Bacto-tryptone, $1 \%$ sodium chloride, $0.5 \%$ yeast extract $\mathrm{pH} 7.5)$. The overnight bacterial cultures are then diluted $1: 100$ in $40 \mathrm{ml}$ of tryptone broth (TB; Becton Dickinson, Milan, Italy) containing $1 \%$ tryptone, $0.5 \% \mathrm{NaCl}$, and grown at $30^{\circ} \mathrm{C}$ and shaken at $150 \mathrm{rpm}$ until an optical density $\mathrm{OD}_{600}=0.4$ is reached, corresponding to a middle-log phase. It has been demonstrated [40] that transferring $E$. coli cultures from Lysogeny broth to TB results in higher average swimming speed. $P$. aeruginosa is grown at $37^{\circ} \mathrm{C}$ and diluted in the same way, using only Lysogeny broth. Bacterial cells are then harvested from culture media by centrifugation $(2200 \mathrm{rpm}$, $5 \mathrm{~min})$ and washed in a prewarmed motility buffer $(10 \mathrm{mM}$ $\mathrm{K}_{2} \mathrm{HPO}_{4} ; 0.1 \mathrm{mM}$ Na-EDTA, pH 7.0; $70 \mathrm{mM} \mathrm{NaCl} ; 0.002 \%$ Tween 20). This process is repeated twice to achieve growth medium depletion and a suitable final bacterial concentration $n_{\text {tot }}$, expressed as colony-forming unit $(\mathrm{CFU}) / \mathrm{ml}$ and calculated from optical density measurements.

The suspensions are diluted 1:1 (vol/vol) with Percoll to match cells and medium buoyant densities [41]. A drop of the 
bacterial suspension is closed between two glass coverslips separated by spacers of various thickness ranging between about 100 and $250 \mu \mathrm{m}$. The coverslips are previously cleaned in ethanol, dried by blowing nitrogen gas, and dipped overnight in a $1 \%(\mathrm{w} / \mathrm{w})$ bovine serum albumin (BSA; Sigma, Milan, Italy) in a water solution. The resulting BSA coating minimizes bacterial adhesion on glass surfaces $[42,43]$. At the end of each measurement, bacterial suspensions are aseptically diluted in warm phosphate buffered saline, plated on Lysogeny agar plates and incubated at $30^{\circ} \mathrm{C}$ (E. coli) or $37^{\circ} \mathrm{C}$ (P. aeruginosa) for at least $16 \mathrm{hr}$ to confirm bacterial vitality and load.

Note that nonmotile, yet alive, bacteria are also present in the suspensions. This loss of motility can be ascribed to several stressful conditions, such as low temperature or low oxygen concentration [44].

\section{A. Experimental setup and imaging}

The sample is imaged in a bright field by an inverted optical microscope (Nikon Eclipse Ti-E) with $100 \times$ magnification (Olympus LUMPlanFL $100 \times, \mathrm{NA}=1$, depth of focus $2 \mu \mathrm{m}$ ) and equipped with a C-MOS camera (Mikrotron EoSens MC1362). We observe a $160 \times 128 \mu \mathrm{m}^{2}$ area of the sample on planes $(x, y)$ parallel to the glass walls [see Fig. 1(c)]. The separation $h$ between top and bottom walls (in the $z$ direction) is scanned every $2 \mu \mathrm{m}$ within $20 \mu \mathrm{m}$ from the walls and every $10 \mu \mathrm{m}$ in the central region, starting from the bottom wall up to the top wall. For each step we acquire a $50 \mathrm{~s}$ time-lapse movie at $2 \mathrm{fps}$, corresponding to 100 frames. The acquired images are analyzed by a custom-made routine to count the number of bacteria for each plane $z$. More precisely, we estimate $N_{\text {tot }}(z)$, which is the total number of bacteria found at a given $z$ value averaged over 100 frames. Swimming cells are too fast to be tracked in three dimensions by means of our setup, therefore we track the nonmotile cells. It is then possible to extract the number of motile bacteria $N\left(z_{i}\right)$ for each $z_{i}$ plane as the difference between the total number of bacteria $N_{\text {tot }}\left(z_{i}\right)$ and the number of nonmotile bacteria. The total fraction of motile bacteria is then estimated by summing over all the planes imaged during the scan: $\varphi=\left[\sum_{i} N\left(z_{i}\right)\right] /\left(\sum_{i} N_{\text {tot }}\left(z_{i}\right)\right]$. Finally, the swimming speed of our $E$. coli and $P$. aeruginosa is evaluated by tracking the motile cells on planes parallel and next to the two walls. In agreement with previous literature $[32,45]$, an average speed of about $30 \mu \mathrm{m} / \mathrm{s}$ and about $35 \mu \mathrm{m} / \mathrm{s}$ is found for E. coli and P. aeruginosa, respectively.

\section{B. Model and simulation settings}

To shed light on the basic mechanisms driving this behavior, we have decided to run numerical simulations that neglect hydrodynamic interactions and consider only steric interactions and Brownian motion. More precisely, the simulations on motile particles confined within a slit rely on a coarse-grained description of the micro-organism cell that neglects its flagella and models its body by a semiflexible chain of $M$ beads, each of diameter $\sigma[46,47]$. By fixing the length scale $\sigma$ and varying $M$, we can consider cells with different anisotropic ratio. Beads belonging to the same cell are kept together by finitely extensible nonlinear elastic (FENE) springs. The rigidity of each cell chain is achieved in terms of a Kratky-Porod potential

$$
U_{K P}=\sum_{i=2}^{M} K\left(1-\frac{\mathbf{r}_{i-1} \cdot \mathbf{r}_{i}}{\left|\mathbf{r}_{i-1}\right|\left|\mathbf{r}_{i}\right|}\right),
$$

where $K$ the bending parameter. Steric interactions between cells are enforced by assuming that beads belonging to different cells interact via a purely repulsive truncated and shifted Lennard-Jones (LJ) potential,

$U_{i, j}^{L J}(r)=\left\{4 \epsilon\left[\left(\frac{\sigma}{r}\right)^{12}-\left(\frac{\sigma}{r}\right)^{6}\right]-V_{L J}\left(r=r_{c}\right)\right\} \theta\left(r-r_{c}\right)$,

where $r=\left|\mathbf{r}_{i}-\mathbf{r}_{j}\right|$ is the distance between the bead pair $(i, j)$ with position, respectively, $\mathbf{r}_{i}$ and $\mathbf{r}_{j}, r_{c}=2^{1 / 6} \sigma$ and $\epsilon$ sets the strength of the interaction. A similar potential $U_{W}$ is used to describe the wall-bead interaction where $r=\left|\mathbf{r}_{i}-\mathbf{r}_{w}\right|$ is the distance between the $i$ th bead and the wall located at $\mathbf{r}_{w}$. In the simulations, the impenetrable walls are located at $z=0$ and $z=h$ while along $x$ and $y$ periodic boundary conditions are assumed. The $i=1$ and the $i=M$ bead are, respectively, the head and tail of the cell, and self-propulsion is obtained by applying at each bead a force of strength $\left|\mathbf{F}_{a}\right|$ along the tail to head direction (cell director). This gives a total force per cell of modulus $M\left|\mathbf{F}_{a}\right|$. Since in our experiments there is always a fraction $1-\varphi$ of cells that are not motile, in the simulations we consider the possibility of having in the system a number $N_{p}$ of bodies for which $F_{a}=0$. If we set $U=U_{L J}+U_{K} P+U_{W}$ as the full potential, the time evolution of the $i$ th bead follows a Langevin equation:

$$
m_{i} \frac{d^{2} \mathbf{r}_{i}}{d t^{2}}=-\gamma \frac{d \mathbf{r}_{i}}{d t}-\nabla_{i} U+\boldsymbol{F}_{a}+\sqrt{2 k_{B} T \gamma} \boldsymbol{\xi}_{i}(t),
$$

where $\gamma$ is the viscous friction felt by each bead, $\nabla_{i}=\frac{\partial}{\partial \mathbf{r}_{i}}, k_{B}$ is the Boltzmann constant, $T$ is the temperature, $m_{i} \stackrel{\equiv}{\equiv} m$ is the mass of the bead, and $\xi_{i}(t)$ is an uncorrelated Gaussian noise with zero mean and unit variance. It is important to notice that the total force acting on each bead in a cell is in general different, in both magnitude and direction, so that each cell rotates due to Eq. (3). Parameters are typically set to lead to strongly overdamped dynamics, which is realistic for microswimmers; the stiffness of the FENE springs is also strong enough that the distance between beads of the same cell is practically constant and equal to $\sigma$; similarly, the value of bending rigidity $K$ is chosen to be sufficiently large to reproduce an effectively rigid cell (see Appendix A for parameter values and mapping to physical units).

The above scheme describes a self-propelled anisotropic particle whose dynamics is essentially governed by the active deterministic force propelling the particle along its tail-head direction and the rotational or translation diffusion due to Brownian motion (see Appendix B). To reproduce the Run and Tumble motility pattern of multiflagellated bacteria such as the wild-type $E$. coli strains, we include in the model the possibility that each body undergoes tumbling from time to time. During a tumbling event, which we consider instantaneous, i.e., of duration $\tau_{T} \simeq d t$, the cell tries to perform a rigid rotation of a given angle $\theta_{T}$ around an axis passing through its center of mass and perpendicular to the cell axis. If the rotated cell 
overlaps with another cell, the move is rejected, otherwise the new position is updated. For simplicity, we fix the value of $\theta_{T}$ to $\sim 70^{\circ}$, which is the expected average tumbling angle for wild-type $E$. coli [30], even though test simulations in which the tumbling direction is chosen from a normally distributed variable (with mean $\sim 70^{\circ}$ and standard deviation $\sim 20^{\circ}$ ) do not show noticeable differences. Between two tumbling events, the cell moves according to Eq. (3) (run). Since the total number of tumbles occurring up to a time $t$ is a Poisson process [7], the distribution of the run duration is expected to be exponential. Hence, each cell attempts to perform a tumbling motion after a run time $T_{\text {run }}$ that is randomly chosen from the distribution

$$
p\left(T_{\text {run }}=t\right)=\frac{1}{\tau_{R}} e^{-t / \tau_{R}}
$$

where the average run time is of the order of $\tau_{R} \sim 10 \tau_{T} \sim 1 \mathrm{~s}$ [30]. For a Run and Reverse motion we simply consider a Run and Tumble move with $\theta_{T}=180^{\circ}$ and the same run time $T_{\text {run }}$. Note that, unlike the numerical model adopted in Ref. [24,25], here the tumbling angle is mostly kept constant while the aspect ratio of the cells is controlled by varying the number of beads $M$ in each cell.

Important dimensionless numbers to consider are the active Péclet number $\mathrm{Pe}=\frac{F_{a} \sigma}{D_{t} \gamma}=\frac{F_{a} \sigma}{k_{B} T}$ (where $D_{t}$ is the translational diffusion coefficient) and the volume fraction, $\phi=$ $\frac{M N \frac{4}{3} \pi\left(\frac{\sigma}{2}\right)^{3}}{V}=\frac{M \pi}{6} \frac{N}{V}$, evaluated in terms of the total number of spheres present in the system. Since we fix $L_{x}=200 \sigma$ and $L_{y}=200 \sigma$, the system volume $V=L_{x} L_{y} h$ may vary with the slab height $h$, and, in order to keep $\phi$ fixed at the experimental value, the total number of cells $N$ in the system has to vary accordingly. All quantities are expressed in terms of the units of mass, length, and energy, which are $m, \sigma$, and $\epsilon$, respectively. The unit for the active force is therefore $\epsilon / \sigma$. As a characteristic simulation time we consider the Brownian time $\tau_{B}=\sigma^{2} / 2 d D_{t}=\gamma \sigma^{2} / 2 d k_{B} T$. The numerical integration of Eq. (3) is performed by using a standard velocity Verlet algorithm with a time step $\delta t=10^{-3} \tau_{B}$. By fixing $\gamma=10$, we set a damping time $\tau_{D}=\tau_{B} / \gamma=0.1 \tau_{B}$. With this choice the inertia term is almost negligible, and, at least for moderate values of the active force, an overdamped description should give similar concentration profiles at the walls.

In order to devise and test a minimal model of motile particles that faithfully reproduces the experimental profiles and that can be used to extend the investigation to conditions not accessible to our experiments, we have simulated a system in which the beads chains are confined within a slit of height $h$ whose value is chosen coherently with the experimental one. For the cell body, we have neglected the contribution of the flagella in cell-cell and cell-wall steric interactions and assumed cells with aspect ratio $1 / 3$, i.e., cells made by $M=3$ beads each. In terms of physical lengths, by assuming $\sigma=$ $0.7 \mu \mathrm{m}$ this would give cells $2.1 \mu \mathrm{m}$ long and $0.7 \mu \mathrm{m}$ wide, in agreement with the sizes of the bacteria in the experiments. The system is initialized by inserting, one by one, in an ordered fashion, a total number $N$ of cells in the simulation box. This initial configuration that, by construction, does not present overlaps between cells is then allowed to equilibrate and spread throughout the system. Once equilibrated, we add a force $F_{a}$ to the fraction $\varphi$ of cells that we want to be motile, and
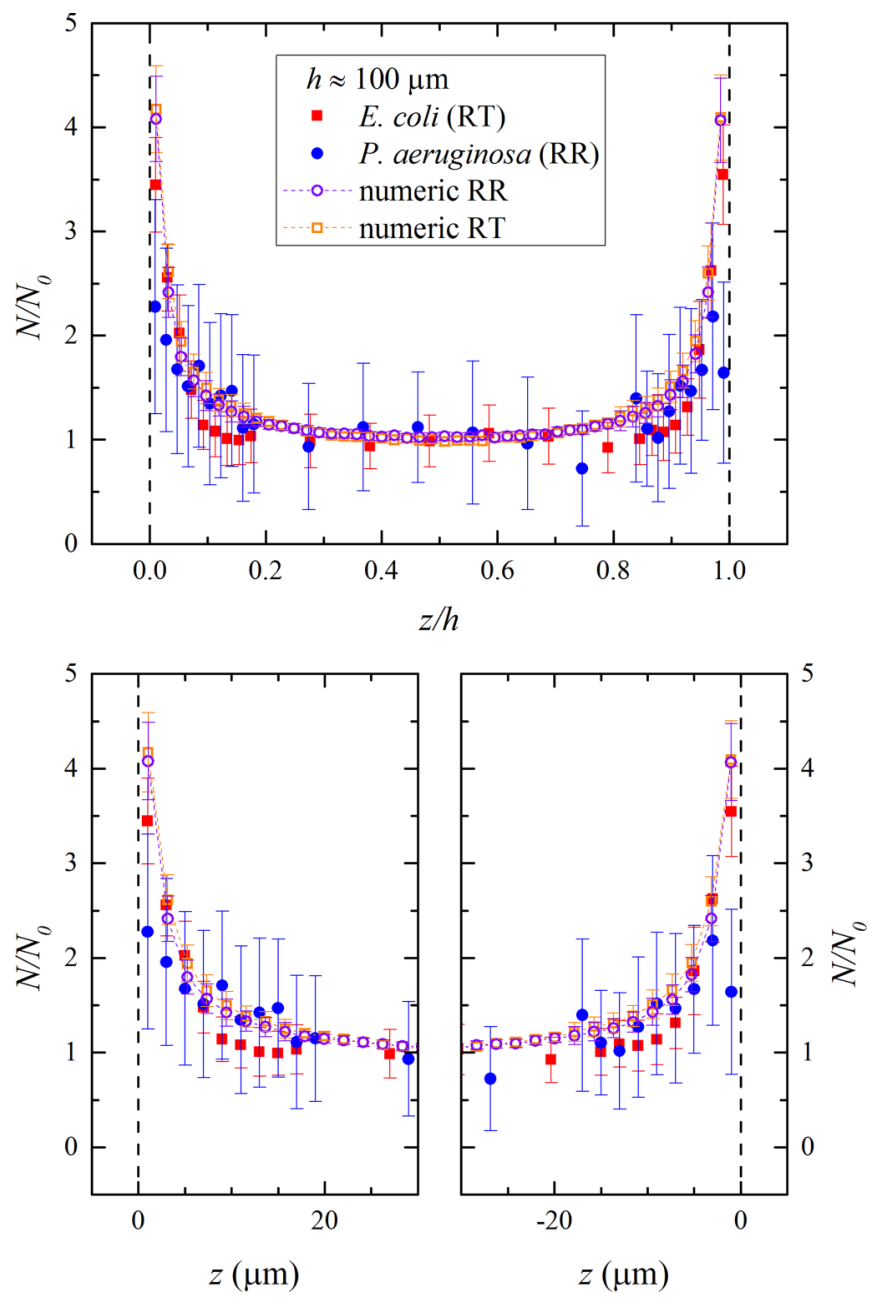

FIG. 2. Top: Experimental concentration profiles obtained for MG1655 E. coli (full squares) and wild-type P. aerugionosa (full circles). The corresponding results from the simulations of the Run and Tumble (RT) (empty squares) and Run and Reverse (RR) (empty circles) models are reported for comparison. In this case the slit height $h \approx 100 \mu \mathrm{m}$ and $75 \%$ of the bacteria are motile. Bottom: Zoom of the concentration profiles near the walls.

its value is set to get an average speed value of the motile cells that is comparable to the one observed experimentally. In our simulations we consider Pe in the range $[150,300]$. This corresponds to self-propelled bodies with an average speed of the order of $[27,50] \mu \mathrm{m} / \mathrm{s}$, i.e., comparable to the ones of the bacteria analyzed in the experiments.

\section{RESULTS}

Figure 2 compares the experimental density profiles obtained for MG1655 E. coli and wild-type P. aeruginosa at $h=100 \mu \mathrm{m}$ with the simulation results for bodies with aspect ratio 1/3 and either Run and Tumble or Run and Reverse motility. Note that the concentration profile is obtained by normalizing the number of motile bacteria at a given height $z, N(z)$, with respect to the average value $N_{0}$ taken away from the walls when the concentration is roughly constant. Some features are worth mentioning: first, although the accumulation peaks for the $P$. aeruginosa are slightly lower with respect 
(a)
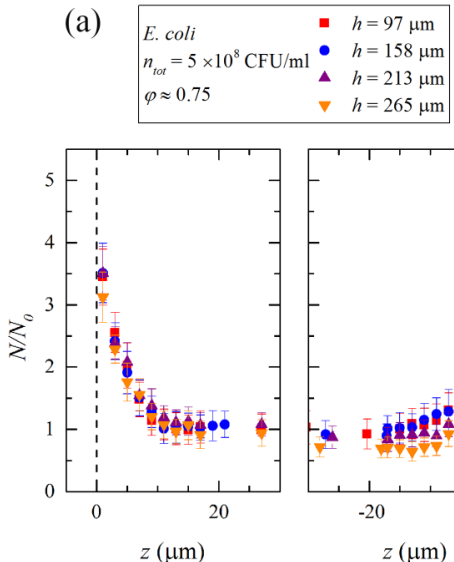

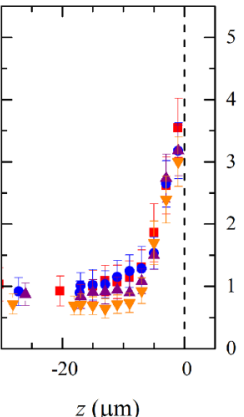

(b)
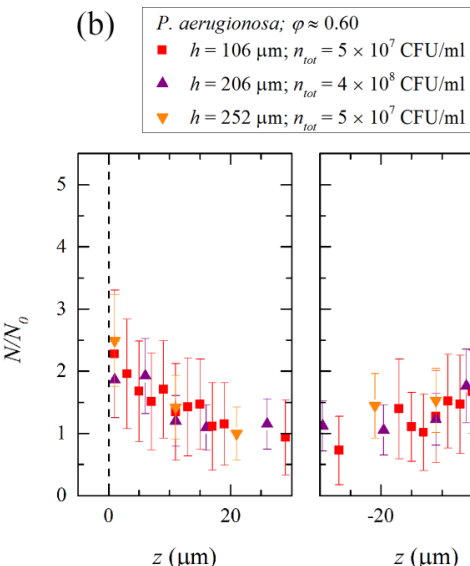

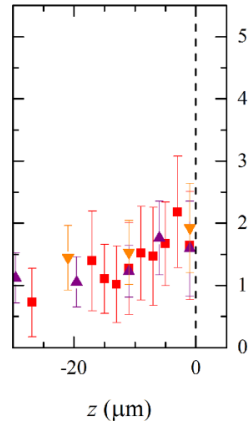

(c)
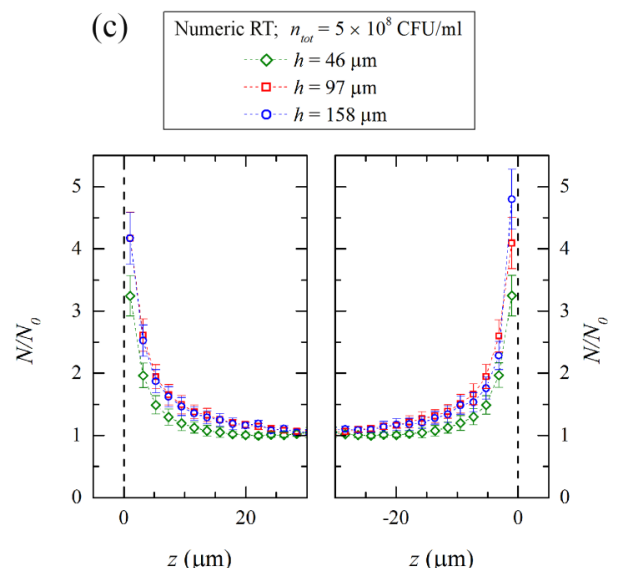

FIG. 3. Concentration profiles for different separations $h$ between the walls in the case of experimentally observed MG1655 E. coli (a) and wild-type $P$. aeruginosa (b) and numerically simulated Run and Tumble (RT) swimmers. The plots report the number of swimming cells $N$ normalized to the average number $N_{0}$ of the central plateau as a function of the distance $z$ from the walls in the near-wall regions.

to the $E$. coli, the concentration profiles of the two species coincide within the statistical errors given by the standard deviations. Second, the simulation results agree nicely with the experimental ones and confirm that the aggregation of bacteria at the walls is significant (about 3.5 higher than the plateau away from the wall; see Fig. 2). Third, the whole concentration profile looks qualitatively independent of the motility pattern (Run and Tumble versus Run and Reverse) considered. This is also confirmed by looking at the distribution of the detention time of the self-propelled cells, namely, the time a cell spends in the layer of width $3 \mu \mathrm{m}$ closest to the wall: the two distributions (i.e., for cells with Run and Tumble and Run and Reverse pattern motility respectively) are rather similar [see Fig. 7(a)].

These results support the view that, although the details of the microscopic dynamics in proximity to the walls can be rather different, they have a negligible impact on the accumulation at the walls, a feature that can be faithfully captured also by simple models of anisotropic motile particles confined in a slit.

\section{A. Effect of the degree of confinement}

The effect of spatial confinement on concentration profile has been studied by changing the height $h$ of the spacer between the two glass coverslips. In Fig. 3 we report the experimental concentration profiles estimated at different slit heights $h$ within the range 100-250 $\mu \mathrm{m}$. Figures 3(a) and 3(b) refer, respectively, to $E$. coli (Run and Tumble) and $P$. aeruginosa (Run and Reverse).

Let us discuss first the behavior of the E. coli [Fig. 3(a)]. As before, the profile of the number of swimming cells $N(z)$ is normalized to the average number $N_{0}$ in the region $\approx 30 \mu \mathrm{m}$ away from the walls. These samples have the same concentration $n_{\text {tot }} \approx 5 \times 10^{8} \mathrm{CFU} / \mathrm{ml}$ and the same fraction of swimming cells $\varphi \approx 0.75$. All profiles exhibit a concentration increase within $20 \mu \mathrm{m}$ from the walls and a horizontal plateau in the central region. The values of the peaks observed in proximity to the walls are about 3.5 times higher than the ones at the plateau.

Figure 3(a) shows that all profiles coincide within error bars independently on the wall separation. The main discrepancy is found in the profile for $h=265 \mu \mathrm{m}$, which displays a topbottom asymmetry, probably due to a mismatch of the buoyant densities. These results are in qualitative agreement with the concentration profiles found for AW405 strain E. coli, which performs a Run and Tumble pattern, reported in Ref. [26], although our experimental profiles show a lower accumulation at the walls. A possible cause of this small discrepancy could be that the bacterial strains used in the two experiments differ either in average velocity or in tumbling frequency.

Similar concentration profiles for the swimming $P$. aeruginosa are presented, for different $h$, in Fig. 3(b). All samples have the same fraction of motile cells $\varphi \approx 0.60$. Irrespective of the slit height $h$, the profiles exhibit a concentration increase within about $20 \mu \mathrm{m}$ from the walls and a horizontal plateau in the central region. The peaks in proximity to the walls are about 2-2.5 times higher than the values observed at the plateau. The error bars for $h=106 \mu \mathrm{m}$ and $h=252 \mu \mathrm{m}$ are larger than the ones for $E$. coli in Fig. 3(b) because of the lower concentration $n_{\text {tot }}=5 \times 10^{7} \mathrm{CFU} / \mathrm{ml}$. As for the $E$. coli, Fig. 3(b) displays profiles that, within error bars, can be considered unaffected by the degree of confinement.

In summary, the experimental findings show that, although these bacteria differ from each other by the swimming pattern, their tendency to accumulate at the walls and, more quantitatively, the overall concentration profile are, at least for the volume fraction considered, similar and independent of $h$. The agreement with the simulations suggests that the aggregation of these species at the walls is mainly due to the interplay of the anisotropic, steric interactions that favor parallel orientation and the random reorientation due either to thermal fluctuations or to tumbling $[24,48]$. Hydrodynamic interactions that, at these concentrations and confinement, do not alter qualitatively the distribution [49] become important at higher concentrations and/or narrow confinement [50,51]. Note that the experiments have been performed for slits heights down to $h \gtrsim 100 \mu \mathrm{m}$. On the other hand, the numerical simulations, which have been extended to slits of height as small as $h=46 \mu \mathrm{m}$ and have smaller statistical errors, suggest a concentration profile that, when plotted as function of the distance $z$ from the wall [Fig. 3(c)], is higher for smaller values of $h$. This is the behavior expected in the ballistic regime where the cell 
travels essentially on straight lines between the two walls [48]. It is important to stress that both in the experiments and in the simulations, although the space available changes with $h$, the concentration of the particles in the system is kept fixed. Hence no effects due to higher concentrations are expected.

\section{B. Effects of speed and anisotropy}

The effects of the cells speed and the cell body anisotropy on the accumulation at the walls cannot be experimentally controlled. Motile cells of different speed are indeed expected to behave differently nearby the walls: more precisely, faster bacteria should accumulate more than slower ones [48,52]. To check this effect, motile Run and Tumbling bodies with different Pe are simulated by keeping all the other parameters fixed. This is achieved by simply keeping the temperature fixed and by properly changing the value of the active force $F_{a}$. As expected, the peak of the cell's concentration at the walls increases as Pe of the system increases [see Fig. 4(a)]. In particular, the accumulation at the walls is twice as large for systems with $\mathrm{Pe}=210\left(v_{0} \sim 27 \mu \mathrm{m} / \mathrm{s}\right)$ and becomes about 3.5 times larger for $\mathrm{Pe}=420\left(v_{0} \sim 50 \mu \mathrm{m} / \mathrm{s}\right)$. In addition, within a distance of almost $20 \mu \mathrm{m}$ from both walls, the higher the Pe, the steeper the accumulation curve.

In our picture cell anisotropy should favor orientation parallel to the walls and hence accumulation. To test this hypothesis numerically, we look at the dependence of the concentration profile on the cells' aspect ratio $w / l$ where $w$ and $l$ are, respectively, the width and the length of the motile body. By keeping fixed the diameter of the beads forming a cell, we have then varied their number $M$ from $M=2$ up to $M=5$, corresponding respectively to aspect ratios from $1 / 2$ to $1 / 5$. In order to highlight only the effect due to anisotropy, the simulations are performed with all the other parameters (i.e., $\phi, \varphi, h$, and $F_{a}$ ) kept fixed. The results are reported in Fig. 4(b): as expected, as $M$ increases (i.e., as the cells become more anisotropic) the aggregation at the walls is extremely enhanced. For instance, for motile cells with aspect ratio $1 / 5$ the peaks developed in proximity to the walls are about six times higher than the constant values (plateau) in the bulk. This behavior is independent of the average speed of the swimmer and confirms that a relevant mechanism in determining the amount of surface aggregation is the anisotropic steric effect that favors a motion of the cell parallel to the wall [48]. Accumulation at the walls has been also analytically predicted in a model of noninteracting spherical active Browian $[53,54]$ and Run and Tumble $[55,56]$ particles. By adapting our model to the limiting case of $M=1$ we confirm wall accumulation also for spherical cells, with a profile that is slightly below the one estimated for $M=2$ (not shown). This picture is further confirmed if one looks at the distribution of the detention times, i.e., the time spent by the cell within the closest layer to the wall (width $3 \mu \mathrm{m}$ ) [see Fig. 7(a)]. Both for the Run and Tumble and Run and Reverse motility pattern the distribution follows an exponential decay with a time scale that is substantially larger than the typical time $\tau_{R}$ for a run of the cell in the bulk. Notice that, as before, since the bead chain concentration is kept fixed at $5 \times 10^{8} \mathrm{ml}^{-1}$ an increase in $M$ corresponds to a decrease of the total number of cells. However, further tests performed with concentrations up to $10^{9} \mathrm{ml}^{-1}$ and $M=5$ (this
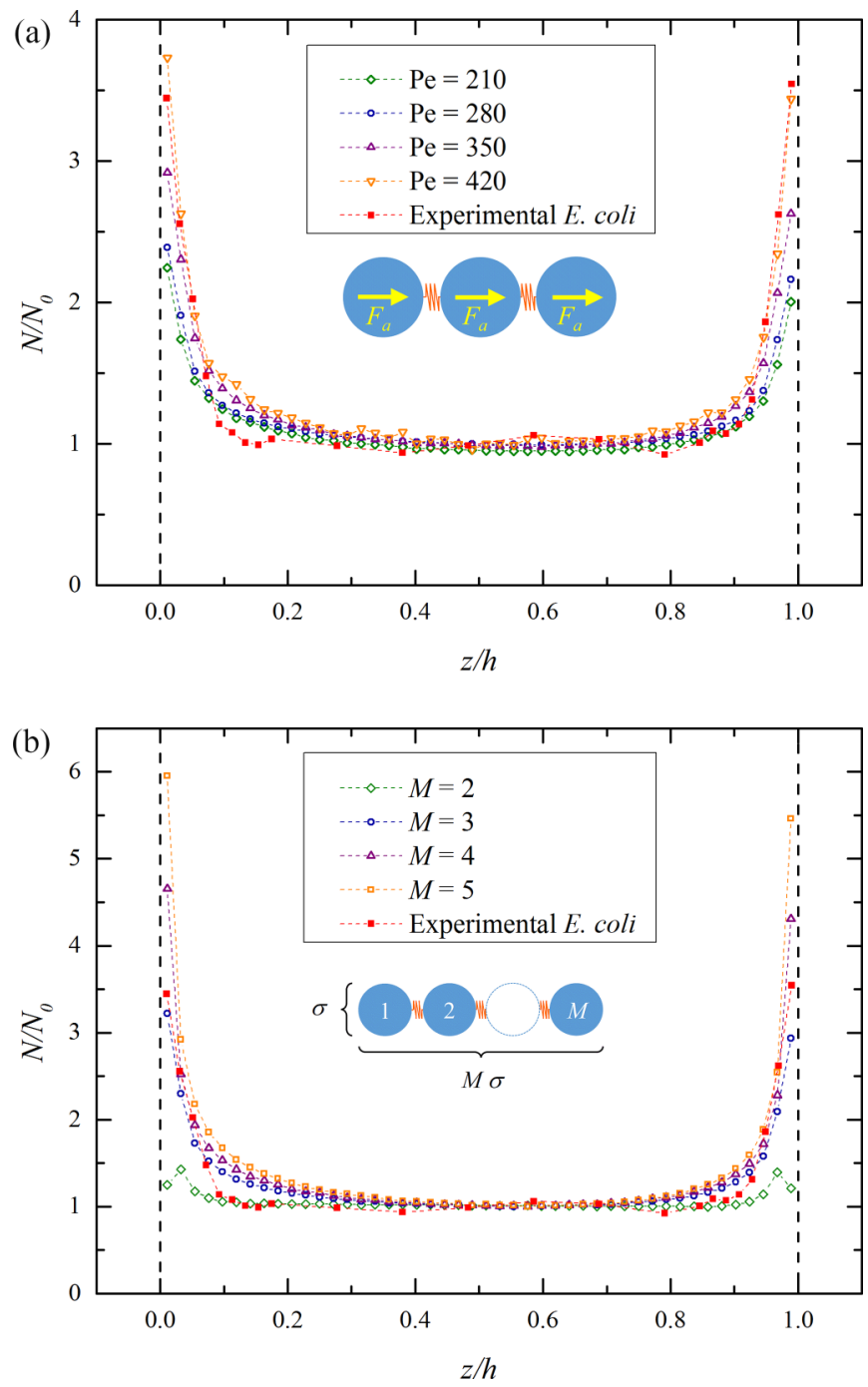

FIG. 4. (a) Concentration profiles of Run and Tumble swimmers confined in a slit of height $h \simeq 100 \mu \mathrm{m}$. Different symbols refer to different values of the active force and hence of the average speed. Note that for higher values of the average speed, the aggregation of the active swimmers near the walls increases. (b) Concentration profile of Run and Tumble swimmers for different body aspect ratio. In the legend $M$ is the number of beads forming a single cell. For all cases, the Péclet number is $\mathrm{Pe}=420$ corresponding to a body average speed of $\sim 50 \mu \mathrm{m} / \mathrm{s}$. The concentration of particles is $\sim 5 \times 10^{8} \mathrm{CFU} / \mathrm{ml}$. The system is confined within a slit of height $h=97 \mu \mathrm{m}$.

gives roughly the same number of cells with $M=3$ ) yield very similar results.

\section{CONCLUSIONS}

We have studied experimentally the concentration profiles of bacterial suspensions confined in slits of heights $h$ that are comparable with the typical persistence length of the cells. In particular, we have considered MG1655 E. coli and wild-type $P$. aerugionosa bacteria, whose motilities are characterized by the Run and Tumble and the Run and Reverse patterns, respectively. The experiments have been completed with numerical simulations of a simple model of self-propelling particles 
whose main ingredients are the anisotropy of the particles and the excluded volume interaction between particles and with the walls. In both experiments and numerical simulations we observe that the concentration profiles are similar regardless of the motility pattern and are characterized by a consistent increase in the number of bacteria within a distance of about $20 \mu \mathrm{m}$ from the walls. This accumulation is an effect of a longer detention time close to the walls which is due to the cells' alignment induced by steric interactions with solid walls. Moreover, the concentration profiles are, within the error bars and for the range of $h$ considered, insensitive to the degree of confinement, due to the relatively low concentration of the bacterial suspensions.

These findings, which confirm previous studies of bacteria aggregation performed on single species [23-26], indicate that, although the microscopic dynamics in proximity to the walls can be rather different, this has a negligible overall impact on the accumulation at the walls, a feature that has been faithfully captured by our simple model of motile cells. Moreover, the fact that our model does not consider long-range hydrodynamic interactions shows that, at least for the density of bacteria considered and for the two mechanisms of motion studied (i.e., Run and Tumble and Run and Reverse), steric interactions with a solid surface and self-propulsion are main features, besides hydrodynamics, that establish the profiles at the walls. Arguably, taking into account the contribution of hydrodynamic effects would result in an even better agreement between experimental and numerical data that could be appreciated in the case of smaller statistical errors. Finally, our simulations show that the number of motile cells at the walls increases both with their average speed and with the aspect ratio of the cell's geometry. For future work, it would be of interest to perform similar joint theoretical and experimental analysis of suspensions of other micro-organisms confined within slits by considering either other motility patterns (e.g., smooth or reverse and flick motion [57]) or cells with different aspect ratios. More important, increasing the bacteria concentration or strongly confining the suspension in microfluidic channels $[58,59]$ would provide useful insights on the interplay between crowding and confinement.

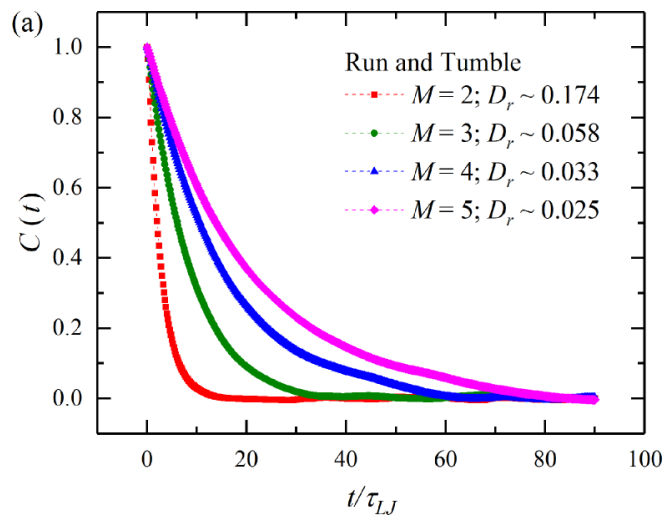

\section{ACKNOWLEDGMENTS}

Grants from the University of Padova No. CPDA138901 and MIUR PRIN 2012NNRKAF are gratefully acknowledged. P.S. and G.J. acknowledge the Cariparo Foundation in the frame of PhD funding action. We are particularly thankful to Prof. Andrea Squartini for providing test bacterial samples, to Dr. Emanuele Locatelli for useful discussions, to Vera Russo for the help in the sample preparation, and to Giulia Colzani and Luca Bonato for support in the data acquisition.

\section{APPENDIX A: SIMULATION DETAILS}

Simulation parameters are fixed by matching the transverse size $\sigma$, the average velocity $v$, and the covered volume $\phi$ of the cells with the corresponding experimental values. For example, by assuming a typical cell width of $\sigma=0.7 \mu \mathrm{m}$ and taking $M=3$, we get a cell of length $2.1 \mu \mathrm{m}$, which is reasonable for the bacteria used in our experiments. The time scale $\tau_{B}=$ $\sigma^{2} / 2 d D_{t}$ can be obtained by matching the diffusion coefficient $D_{t}$ with the ones expected for a passive cell made by $M$ spheres. Since $D_{t}=k_{B} T / \gamma$ and, to a crude approximation neglecting the anisotropic shape of the cell, $\gamma=3 M \pi \eta \sigma$, we have

$$
\tau_{B}=\frac{3 M \pi \sigma^{3} \eta}{2 d k_{B} T} .
$$

By using the nominal water viscosity $\eta=1 \mathrm{cP}=10^{-3} \mathrm{~kg} / \mathrm{ms}$ and assuming $\sigma \sim 0.7 \mu \mathrm{m}, k_{B} T=4.11 \times 10^{-21} \mathrm{~J}$, we get a diffusion coefficient on the order of

$$
\begin{aligned}
D_{t} & =\frac{k_{B} T}{3 M \pi \eta \sigma} \\
& =\frac{4.11 \times 10^{-21} \mathrm{~kg} \mathrm{~m}^{2} / \mathrm{s}^{2}}{3 M \pi \times 10^{-3} \mathrm{~kg} / \mathrm{ms} \times 0.7 \times 10^{-6} \mathrm{~m}} \\
& \sim \frac{0.62 \mu \mathrm{m}^{2}}{M \mathrm{~s}} .
\end{aligned}
$$

This gives a Brownian time on the order of $\tau_{B} \sim M \times 0.13$ $\mathrm{s}=0.39 \mathrm{~s}$ in $d=3$. Since the average velocities of the cells are given in units of $\sigma / \tau_{B}$, in order to reproduce typical bacteria velocities $v \sim 20-50 \mu \mathrm{m} / \mathrm{s}$ [which, in simulation

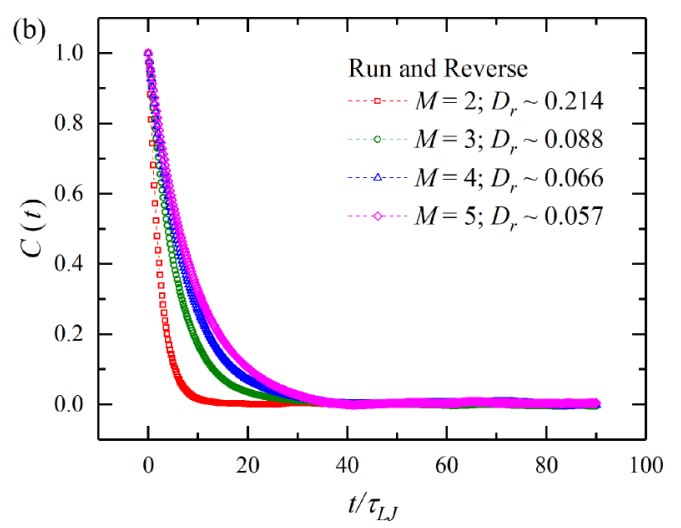

FIG. 5. (a) Velocity-velocity correlation function $C(t)$ for Run and Tumble swimmers (a) and Run and Reverse swimmers (b) obtained for several values of the number of beads $M$. Time is given in units of the characteristic time of the Lennard-Jones interaction $\tau_{L J}=\sigma(m / \epsilon)^{2}$. By fitting the curves by an exponential, the values of $D_{r}$ can be obtained. The higher the anisotropy, the smaller the decay rate, hence the value of $D_{r}$. Data refer to a collection of Run and Tumble swimmers where $F_{a}=300$ and concentration is $5 \times 10^{7} \mathrm{ml}^{-1}$. Data refer to a collection swimmers with $F_{a}=300$ and concentration $5 \times 10^{7} \mathrm{ml}^{-1}$. 


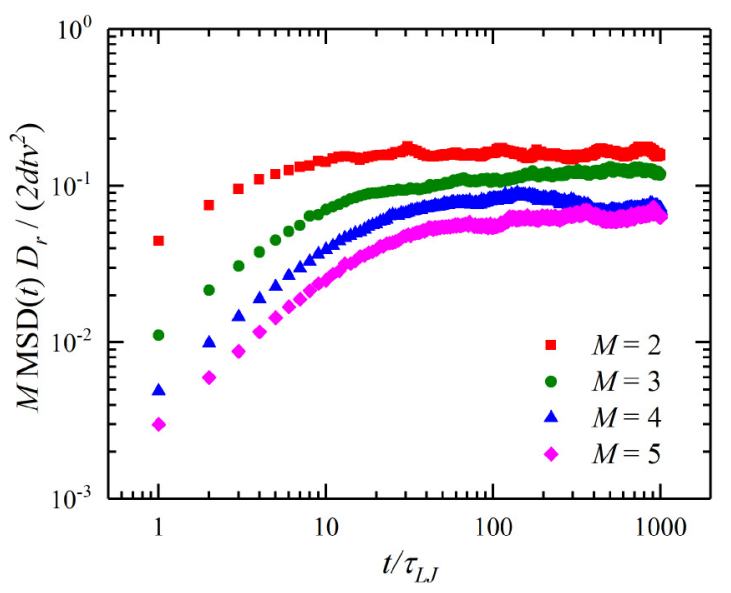

FIG. 6. Time dependence of the ratio $M \operatorname{MSD}(t) D_{r} /\left(2 d t v^{2}\right)$, where $\operatorname{MSD}(t)=\left\langle[\mathbf{r}(t)-\mathbf{r}(0)]^{2}\right\rangle$, for Run and Tumble cells. Different curves refer to different values of the number of beads $M$ forming each cell. Data refer to a collection swimmers with $F_{a}=300$ and concentration $5 \times 10^{7} \mathrm{ml}^{-1}$.

units, reads $\left.v \sim(4-10) M \sigma / \tau_{B}\right]$, one has to consider active forces of the order $F_{a}=\gamma v=10 \times(4-10) M \sigma / \tau_{B}^{2}=$ (40-100) $M \sigma / \tau_{B}^{2}$. We note that $v$ is the speed of each bacterium, whereas $F_{0}$ is the force applied to each bead of a single bacterium.

Note that, due to the low particle concentration (approximately $5 \times 10^{8} \mathrm{ml}^{-1}$ ), repulsive interactions between cells are rare. Indeed, by switching off the Lennard-Jones repulsion, the concentration profiles remain unaltered through the whole system. By neglecting the two-body interactions one can make simulations faster and better highlight the role played by other physical parameters, such as the speed and anisotropy of the cells on their accumulation at the walls.

\section{APPENDIX B: ESTIMATE OF $D_{r}$ AND $D_{t}$ FROM SIMULATIONS}

For the set of active cells in the system, the mean-squared displacement of the center of mass $\mathbf{r}_{C M}=M^{-1} \sum_{i}^{M} \mathbf{r}_{i}$ of each

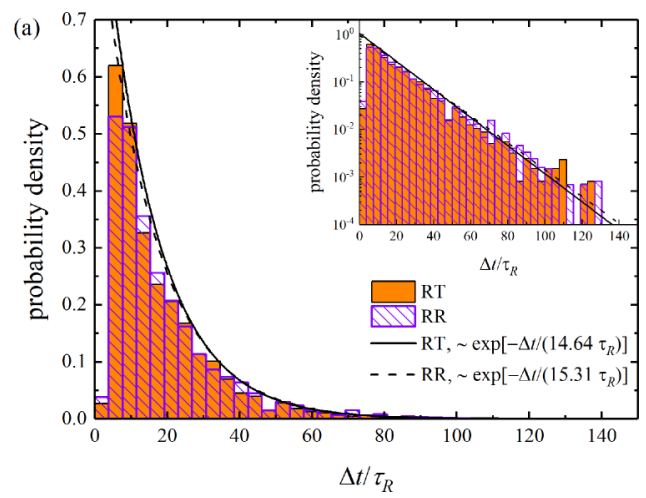

cell depends also on the active force $F_{a}$. For sufficiently long times it is given by

$$
\left\langle\left[\mathbf{r}_{C M}(t)-\mathbf{r}_{C M}(0)\right]^{2}\right\rangle=2 d D_{t} \frac{v^{2}}{D_{r}} t .
$$

Note that the rotational diffusion constant $D_{r}$ appears in Eq. (B1). This can be estimated by computing, for instance, the velocity-velocity correlation function

$$
C(0, t)=\left\langle\mathbf{v}_{C M}(0) \cdot \mathbf{v}_{C M}(t)\right\rangle,
$$

where $\langle\ldots\rangle$ denotes an average over the number of active particles. This quantity represents a measure of the persistence of the cell's trajectory. For constant speed, this function can be computed by looking at the correlation function of the angle $\alpha(t)$ describing the head-tail orientation of the cell during its motion:

$$
C(0, t)=v_{C M}^{2}\langle\cos [\alpha(0)-\alpha(t)]\rangle .
$$

This is reported in Fig. 5 for cells with different values of $M$ (i.e., different anisotropy). As expected each curve displays an exponential decay $C(t)=\exp \left(-2 D_{r} t\right)$ with the estimate of $D_{r}$ reported in the legend for each value of $M$. One can see that $D_{r}$ is larger for less anisotropic cells, although the Run and Reverse one is from 1.5 to 2 times larger than the corresponding Run and Tumble one. This result suggests that the former reorient faster than the latter, even though, once more, this effect has negligible consequences on both concentration profiles. Finally a plot of $M\left\langle[\mathbf{r}(t)-\mathbf{r}(0)]^{2}\right\rangle D_{r} / 2 d t v^{2}$ versus time for active cells is reported in Fig. 6. At late times, the curves reach a constant value that we identify with the estimate of $M D_{t}$. Note that, as $M$ increases, this value approaches a common value confirming the $1 / M$ dependence of $D_{t}$ also for the late time behavior of the active cells.

\section{APPENDIX C: DETENTION TIME}

In order to check in more detail whether the motility pattern influences the accumulation of active cells at the walls, we have calculated the detention time. This is defined as the time interval $\Delta t$ a cell spends within the layer of thickness $h=3 \mu \mathrm{m}$ closest to the wall (outer layer). More specifically,

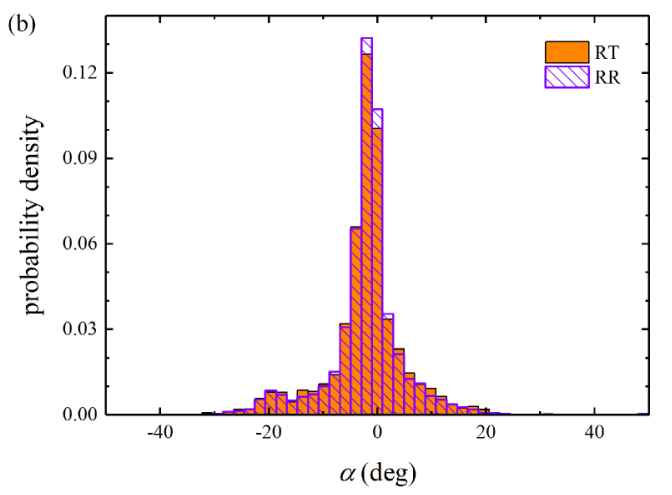

FIG. 7. (a) Detention time distribution of Run and Tumble (RT) and Run and Reverse (RR) swimmers in the layer of width $\Delta z=3 \mu \mathrm{m}$ closest to the wall (outer layer). Note that time is given in units of the average run time $\tau_{R}$. Both distributions display an exponential decay whose time scale has been estimated by an exponential fit (see values in the legend). (b) Distribution of the angle $\alpha$ between the cell axis and the wall for Run and Tumble (RT) and Run and Reverse (RR) swimmers near a wall. Data refer to a collection of swimmers where $F_{a}=200$ and concentration is $5 \times 10^{8} \mathrm{ml}^{-1}$. 
for each trajectory we monitor the times $t_{i}$ and $t_{f}$ at which each cell respectively enters and leaves the layer and compute the residence time $\Delta t=t_{f}-t_{i}$. For a given simulation, this event occurs several times, and we collect all the $\Delta t$ of a cell in an histogram. The final histogram is obtained by summing over all cells. In Fig. 7(a) we report the normalized histogram of detention times for systems with Run and Tumble (red) and Run and Reverse (black) motility mechanisms. For both motility patterns, the distribution of detention times decays exponentially with a time scale of the order of $14 \tau_{R}$ for Run and Tumble and $15 \tau_{R}$ for cells following a Run and Reverse motion. This is a clear indication that, close to the wall, the cells, irrespective of their motility pattern, tend to persist in their motion parallel to the wall for periods that are longer than those characterizing the straight runs in the bulks. Although the difference is very small, it is interesting to notice that cells with Run and Reverse motion tend to stay longer along the wall. This is somehow expected since reverting the motion does not affect its direction, which is changed by random fluctuations. One can also look at the typical direction of the cell motion while it is visiting the outer layers. This can be estimated by monitoring the angle $\alpha$ that the major axis of the cell forms with the wall. The corresponding histogram, averaged over all cells, is reported in Fig. 7(b): the normalized distributions for Run and Tumble and Run and Reverse cells are again rather similar and characterized by a pronounced peak at $\alpha \simeq 0^{\circ}$. This confirms that the cells preferentially move parallel to the surface, irrespective of their mobility pattern. The two distributions decay very rapidly to zero, and cells that deviate from the wall direction more than $\simeq 40^{\circ}$ are very rare.
[1] S. Ramaswamy, The mechanics and statistics of active matter, Annu. Rev. Condens. Matter Phys. 1, 323 (2010).

[2] D. Saintillan and M. J. Shelley, Theory of active suspensions, in Complex Fluids in Biological Systems (Springer, New York, 2015), pp. 319-355.

[3] E. M. Purcell, Life at low Reynolds number, Am. J. Phys. 45, 3 (1977).

[4] E. Lauga, Life around the scallop theorem, Soft Matter 7, 3060 (2011).

[5] M. C. Marchetti, J. F. Joanny, S. Ramaswamy, T. B. Liverpool, J. Prost, M. Rao, and R. A. Simha, Hydrodynamics of soft active matter, Rev. Mod. Phys. 85, 1143 (2013).

[6] J. Elgeti, R. G. Winkler, and G. Gompper, Physics of microswimmers-single particle motion and collective behavior: A review, Rep. Prog. Phys. 78, 056601 (2015).

[7] H. C. Berg, Random Walks in Biology (Princeton University Press, Princeton, 1993).

[8] M. E. Cates, Diffusive transport without detailed balance in motile bacteria: Does microbiology need statistical physics? Rep. Prog. Phys. 75, 042601 (2012).

[9] K. Drescher, J. Dunkel, L. H. Cisneros, S. Ganguly, and R. E. Goldstein, Fluid dynamics and noise in bacterial cell-cell and cell-surface scattering, Proc. Natl. Acad. Sci. USA 108, 10940 (2011).

[10] W. C. K. Poon, From Clarkia to Escherichia and Janus: The physics of natural and synthetic active colloids, in International School of Physics Enrico Fermi, Proceedings of the International School of Physics Enrico Fermi, edited by C. Bechinger, F. Sciortino, and P. Ziherl, Vol. 184 (IOS, Amsterdam, 2013), pp. 317-386.

[11] J. Stenhammar, D. Marenduzzo, R. J. Allen, and M. E. Cates, Phase behaviour of active Brownian particles: The role of dimensionality, Soft Matter 10, 1489 (2014).

[12] C. Y. Kao, W. H. Lin, C. C. Tseng, A. B. Wu, M. C. Wang, and J. J. Wu, The complex interplay among bacterial motility and virulence factors in different Escherichia coli infections, Eur. J. Clin. Microbiol. Infect. Dis. 33, 2157 (2014).

[13] J. Maennik, R. Driessen, P. Galajda, J. E. Keymer, and C. Dekker, Bacterial growth and motility in sub-micron constrictions, Proc. Natl. Acad. Sci. USA 106, 14861 (2009).
[14] P. Galajda, J. Keymer, P. Chaikin, and R. Austin, A wall of funnels concentrates swimming bacteria, J. Bacteriol. 189, 8704 (2007).

[15] M. Contino, E. Lushi, I. Tuval, V. Kantsler, and M. Polin, Microalgae Scatter off Solid Surfaces by Hydrodynamic and Contact Forces, Phys. Rev. Lett. 115, 258102 (2015).

[16] G. Volpe, I. Buttinoni, D. Vogt, H.-J. Kümmerer, and C. Bechinger, Microswimmers in patterned environments, Soft Matter 7, 8810 (2011).

[17] I. Berdakin, Y. Jeyaram, V. V. Moshchalkov, L. Venken, S. Dierckx, S. J. Vanderleyden, A. V. Silhanek, C. A. Condat, and V. I. Marconi, Influence of swimming strategy on microorganism separation by asymmetric obstacles, Phys. Rev. E 87, 052702 (2013).

[18] C. Bechinger, R. Di Leonardo, H. Löwen, C. Reichhardt, G. Volpe, and G. Volpe, Active particles in complex and crowded environments, Rev. Mod. Phys. 88, 045006 (2016).

[19] E. Lauga, W. R. DiLuzio, G. M. Whitesides, and H. A. Stone, Swimming in circles: Motion of bacteria near solid boundaries, Biophys. J. 90, 400 (2006).

[20] W. R. DiLuzio, L. Turner, M. Mayer, P. Garstecki, D. B. Weibel, H. C. Berg, and G. M. Whitesides, Escherichia coli swim on the right-hand side, Nature (London) 435, 1271 (2005).

[21] R. Di Leonardo, D. Dell'Arciprete, L. Angelani, and V. Iebba, Swimming with an Image, Phys. Rev. Lett. 106, 038101 (2011).

[22] L. Rothschild, Non-random distribution of bull spermatozoa in a drop of sperm suspension, Nature (London) 198, 1221 (1963).

[23] A. P. Berke, L. Turner, H. C. Berg, and E. Lauga, Hydrodynamic Attraction of Swimming Microorganisms by Surfaces, Phys. Rev. Lett. 101, 038102 (2008).

[24] G. Li and J. X. Tang, Accumulation of Microswimmers Near a Surface Mediated by Collision and Rotational Brownian Motion, Phys. Rev. Lett. 103, 078101 (2009).

[25] G. Li, J. Bensson, L. Nisimova, D. Munger, P. Mahautmr, J. X. Tang, M. R. Maxey, and Y. V. Brun, Accumulation of swimming bacteria near a solid surface, Phys. Rev. E 84, 041932 (2011).

[26] M. Molaei, M. Barry, R. Stocker, and J. Sheng, Failed Escape: Solid Surfaces Prevent Tumbling of Escherichia Coli, Phys. Rev. Lett. 113, 068103 (2014). 
[27] O. Sipos, K. Nagy, R. Di Leonardo, and P. Galajda, Hydrodynamic Trapping of Swimming Bacteria by Convex Walls, Phys. Rev. Lett. 114, 258104 (2015).

[28] S. Bianchi, F. Saglimbeni, and R. Di Leonardo, Holographic imaging reveals the mechanism of wall entrapment in swimming bacteria, Phys. Rev. X 7, 011010 (2017).

[29] I. D. Vladescu, E. J. Marsden, J. Schwarz-Linek, V. A. Martinez, J. Arlt, A. N. Morozov, D. Marenduzzo, M. E. Cates, and W. C. K. Poon, Filling an Emulsion Drop with Motile Bacteria, Phys. Rev. Lett. 113, 268101 (2014).

[30] H. C. Berg, E. coli in Motion (Springer Science \& Business Media, New York, 2008).

[31] V. A. Martinez, R. Besseling, O. A. Croze, J. Tailleur, M. Reufer, J. Schwarz-Linek, L. G. Wilson, M. A. Bees, and W. C. Poon, Differential dynamic microscopy: A high-throughput method for characterizing the motility of microorganisms, Biophys. J. 103, 1637 (2012).

[32] R. Stocker, Reverse and flick: Hybrid locomotion in bacteria, Proc. Natl. Acad. Sci. USA 108, 2635 (2011).

[33] H. C. Berg and R. A. Anderson, Bacteria swim by rotating their flagellar filaments, Nature (London) 245, 380 (1973).

[34] H. C. Berg and D. A. Brown, Chemotaxis in Escherichia coli analysed by three-dimensional tracking, Nature (London) 239, 500 (1972).

[35] M. T. Madigan, J. M. Martinko, D. A. Stahl, and D. P. Clark, Brock Biology of Microorganisms (Benjamin Cummings, San Francisco, 2010).

[36] P. D. Frymier, R. M. Ford, H. C. Berg, and P. T. Cummings, Three-dimensional tracking of motile bacteria near a solid planar surface, Proc. Natl. Acad. Sci. USA 92, 6195 (1995).

[37] N. Dasgupta, M. C. Wolfgang, A. L. Goodman, S. K. Arora, J. Jyot, S. Lory, and R. Ramphal, A four-tiered transcriptional regulatory circuit controls flagellar biogenesis in Pseudomonas aeruginosa, Mol. Microbiol. 50, 809 (2003).

[38] C. Qian, C. C. Wong, S. Swarup, and K.-H. Chiam, Bacterial tethering analysis reveals a "run-reverse-turn" mechanism for pseudomonas species motility, Appl. Environ. Microbiol. 79, 4734 (2013).

[39] C. S. Barker, B. M. Pruss, and P. Matsumura, Increased motility of Escherichia coli by insertion sequence element integration into the regulatory region of the $f h D$ operon, J. Bacteriol. 186, 7529 (2004).

[40] J. Schwarz-Linek, J. Arlt, A. Jepson, A. Dawson, T. Vissers, D. Miroli, T. Pilizota, V. A. Martinez, and W. C. K. Poon, Escherichia coli as a model active colloid: A practical introduction, Colloids Surfaces B 137, 2 (2016).

[41] H. M. López, J. Gachelin, C. Douarche, H. Auradou, and E. Clément, Turning Bacteria Suspensions into Superfluids, Phys. Rev. Lett. 115, 028301 (2015).

[42] E. Bulard, M.-P. Fontaine-Aupart, H. Dubost, W. Zheng, M.-N. Bellon-Fontaine, J.-M. Herry, and B. Bourguignon, Competition of bovine serum albumin adsorption and bacterial adhesion onto surface-grafted ODT: In situ study by vibrational SFG and fluorescence confocal microscopy, Langmuir 28, 17001 (2012).

[43] M. C. M. Vanloosdrecht, W. Norde, J. Lyklema, and A. J. B. Zehnder, Hydrophobic and electrostatic parameters in bacterial adhesion, Aquatic Sci. 52, 103 (1990).

[44] T. Winther, L. Xu, K. Berg-Sørensen, S. Brown, and L. B. Oddershede, Effect of energy metabolism on protein motility in the bacterial outer membrane, Biophys. J. 97, 1305 (2009).

[45] T. B. Doyle, A. C. Hawkins, and L. L. McCarter, The complex flagellar torque generator of Pseudomonas aeruginosa, J. Bacteriol. 186, 6341 (2004).

[46] C. Valeriani, M. Li, J. Novosel, J. Arlt, and D. Marenduzzo, Colloids in a bacterial bath: Simulations and experiments, Soft Matter 7, 5228 (2011).

[47] A. Suma, G. Gonnella, D. Marenduzzo, and E. Orlandini, Motility-induced phase separation in an active dumbbell fluid, Europhys. Lett. 108, 56004 (2014).

[48] J. Elgeti and G. Gompper, Self-propelled rods near surfaces, Europhys. Lett. 85, 38002 (2009).

[49] J. P. Hernandez-Ortiz, P. T. Underhill, and M. D. Graham, Dynamics of confined suspensions of swimming particles, J. Phys.: Condens. Matter 21, 204107 (2009).

[50] J. P. Hernandez-Ortiz, C. G. Stoltz, and M. D. Graham, Transport and Collective Dynamics in Suspensions of Confined Swimming Particles, Phys. Rev. Lett. 95, 204501 (2005).

[51] P. T. Underhill, J. P. Hernandez-Ortiz, and M. D. Graham, Diffusion and Spatial Correlations in Suspensions of Swimming Particles, Phys. Rev. Lett. 100, 248101 (2008).

[52] J. Elgeti and G. Gompper, Wall accumulation of self-propelled spheres, Europhys. Lett. 101, 48003 (2013).

[53] G. C. Wagner, F. M. Hagan, and A. Baskaran, Steady-state distributions of ideal active Brownian particles under confinement and forcing, J. Stat. Mech.: Theor. Exp. 2017, 043203 (2017).

[54] A. Dugzun and V. J. Selinger, Active Brownian particles near straight or curved walls: Pressure and boundary layers, arXiv:1712.09627 (2017).

[55] J. Elgeti and G. Gompper, Run-and-tumble dynamics of selfpropelled particles in confinement, Europhys. Lett. 109, 58003 (2015).

[56] B. Ezhilan, R. Alonso-Matilla, and D. Saintillan, On the distribution and swim pressure of run-and-tumble particles in confinement, J. Fluid Mech. 781 (2015).

[57] L. Xie, T. Altindal, S. Chattopadhyay, and X.-L. Wu, Bacterial flagellum as a propeller and as a rudder for efficient chemotaxis, Proc. Natl. Acad. Sci. USA 108, 2246 (2011).

[58] E. Locatelli, F. Baldovin, E. Orlandini, and M. Pierno, Active Brownian particles escaping a channel in single file, Phys. Rev. E 91, 022109 (2015).

[59] E. Locatelli, M. Pierno, F. Baldovin, E. Orlandini, Y. Tan, and S. Pagliara, Single-File Escape of Colloidal Particles from Microfluidic Channels, Phys. Rev. Lett. 117, 038001 (2016). 